\section{Cicatricial changes in ocular pemphigus}

P Chirinos-Saldaña ${ }^{1}$, I Zuñiga-Gonzalez'

JC Hernandez-Camarena ${ }^{1}$, A Navas ${ }^{1}$,

T Ramirez-Luquin ${ }^{1}$, A Robles-Contreras², MC Jimenez-Martinez ${ }^{2,3}$, A Ramirez-Miranda?, VM Bautista-de Lucio ${ }^{4}$ and EO Graue-Hernandez ${ }^{1}$

Keywords: ocular pemphigus; conjunctival scarring; drug-induced pemphigus; pemphigus vulgaris characteristics of ocular involvement in patients with pemphigus at an ophthalmological referral center. Methods A retrospective review was conducted on patients with the immunopathological diagnosis of pemphigus examined between 1 January 2000 and 1 April 2010. Uncorrected distance visual acuity (UDVA), best corrected distance visual acuity (BCVA), ocular symptoms, and ocular surface inflammatory and scarring changes were assessed.

Results A total of 15 patients were identified, with a mean age of $68.27 \pm 14.35$ years, and $80 \%(n=12)$ were female. Extraocular involvement was reported in one patient. All of the eyes showed cicatricial changes in the conjunctiva. In all, 6 eyes (20\%) were classified as stage $I ; 12$ eyes (40\%) as stage II; 10 eyes (33\%) as stage III; and 2 eyes $(7 \%)$ as stage IV. A statistically significant association was found between BCVA and the severity of ocular involvement. The mean BCVA logMAR was 1.66 (20/914), with a range from logMAR $0(20 / 20)$ to logMAR 4 (NLP). Other ocular diseases were found in $8(53.3 \%)$, systemic diseases in 10 $(66.7 \%)$, and the use of pemphigus-inducing drugs in 10 patients $(66.7 \%)$.

Conclusions The present report represents the largest series of ocular involvement in pemphigus confirmed by immunopathology. The clinical manifestations varied from conjunctival hyperemia to corneal scarring and perforation. There was a strong association between scarring changes and low BCVA. Ocular and systemic diseases as well as the use of pemphigus-inducing drugs may predispose to ocular cicatricial changes observed in this series.

Eye (2014) 28, 459-465; doi:10.1038/eye.2014.3; published online 31 January 2014

\section{Introduction}

The term 'pemphigus' includes a group of chronic autoimmune bullous diseases that involve acantholysis and blisters of the epidermis. The major variants of pemphigus include pemphigus vulgaris (PV), pemphigus foliaceus (PF), paraneoplastic pemphigus, and drug-related pemphigus. ${ }^{1}$ The most common pemphigus form is PV, which affects the skin and mucus membranes with an incidence that varies from 0.42 to 1.6 cases per 100000 individuals; it is more frequent among Ashkenazi Jews. ${ }^{2}$ It typically appears in the fourth or fifth decade of life, particularly in women (66 vs 44\%, for women and men, respectively). 3,4 Typically, PV is characterized by suprabasal acantholysis and immunoglobulin G (IgG) and C3 deposits in the intercellular substance, evidenced by direct immunofluorescence or indirect immunofluorescence, with the appearance of a honeycomb. ${ }^{5}$ Diagnosis with immunofluorescence techniques has a sensitivity of 90-100\%.

Traditionally, it has been described that ocular involvement is uncommon in PV. Previous studies have shown that the most common ocular presentation is non-cicatricial conjunctivitis, ${ }^{6-9}$ and in rare cases, erosions on the eyelids and the conjunctiva are observed. However, some cases of 'atypical' presentation characterized by conjunctival scarring and corneal perforation have also been reported. ${ }^{10-12}$ In contrast, ocular mucous membrane pemphigoid (MMP) presents as progressive cicatrizing conjunctivitis, which if left untreated, results in scarring and obliteration of the conjunctival fornices (symblepharon), ocular
${ }^{1}$ Cornea and Refractive Surgery Department, Institute of Ophthalmology 'Fundación de Asistencia Privada Conde de Valenciana', Mexico City, Mexico

${ }^{2}$ Immunology Department, Research Unit Institute, Institute of Ophthalmology 'Fundación de Asistencia Privada Conde de Valenciana', Mexico City, Mexico

${ }^{3}$ Immunology Lab, Department of Biochemistry, Faculty of Medicine, National Autonomous University of Mexico, Mexico City, Mexico

${ }^{4}$ Microbiology and Ocular Proteomics Department, Research Unit, Institute of Ophthalmology 'Fundación de Asistencia Privada Conde de Valenciana', Mexico City, Mexico

Correspondence:

EO Graue-Hernandez, Instituto de Oftalmología Conde de Valenciana, Chimalpopoca No 14, Col. Obrera, Mexico City CP 06800, Mexico.

Tel: +52 55 55206720; Fax: +52 55544216700 .

E-mail: egraueh@gmail.com

Received: 10 December 2012

Accepted in revised form:

9 December 2013

Published online:

31 January 2014 
surface disease, and corneal ulceration and scarring. Similar to pemphigus, it is an immune-mediated disease, but it is characterized by deposits of autoantibodies on the basement membrane zone at the epithelialsubepithelial junction of mucus membranes and occasionally the skin.

The purpose of the present study was to describe the atypical clinical characteristics of patients with confirmed immunopathological diagnoses of pemphigus, who presented to the practice with ocular cicatricial changes simulating ocular MMP

\section{Materials and methods}

We performed a retrospective review of clinical records of patients with PV diagnosed by histopathology and immunofluorescence of the conjunctiva at the Institute of Ophthalmology 'Fundación Conde de Valenciana' from January 2000 to December 2011. The internal review board and the ethics committee approved this study protocol. Patient age, sex, distribution of extraocular lesions, ocular signs (redness, conjunctival blisters, subconjunctival fibrosis, fornix foreshortening, symblepharon, corneal opacities and persistent epithelial defects, perforation, entropion, ectropion, and trichiasis), and ocular symptoms (dryness, foreign body sensation, pain, or discomfort) as well as uncorrected distance visual acuity (UDVA), best corrected distance visual acuity (BCVA), and intraocular pressure were recorded.
Concomitant systemic and/or ocular pathology and previous systemic or ocular medications were also documented.

The severity of the disease was graded according to the classification by Foster $\mathrm{et}^{\mathrm{al}}{ }^{13}$ as follows: stage I, conjunctival inflammation, mucus discharge, small patches of rose bengal-stained conjunctival epithelium, and fine conjunctival subepithelial fibrosis; stage II, foreshortening of the inferior conjunctival cul-de-sac; stage III, symblepharon formation; and stage IV, severe sicca syndrome, ocular surface keratinization, and ankyloblepharon.

Statistical analysis was performed using SPSS v.17 (IBM Corporation, Armonk, NY, USA). Descriptive statistics and Pearson's correlation were used to assess the symmetry of ocular involvement.

\section{Results}

Initially, we identified the clinical records of 57 patients with cicatricial conjunctivitis diagnoses. Of those, 15 patients $(26.31 \%)$ had immunopathological diagnoses of pemphigus (Figure 1a) and were, therefore, included in this report. There were not specialized ophthalmological center at the origin place of $80 \%$ of the patients in our series.

The mean time between initial presentation and conjunctival biopsy was $9.69 \pm 8.78$ weeks ( $R:$ 2-35 weeks).
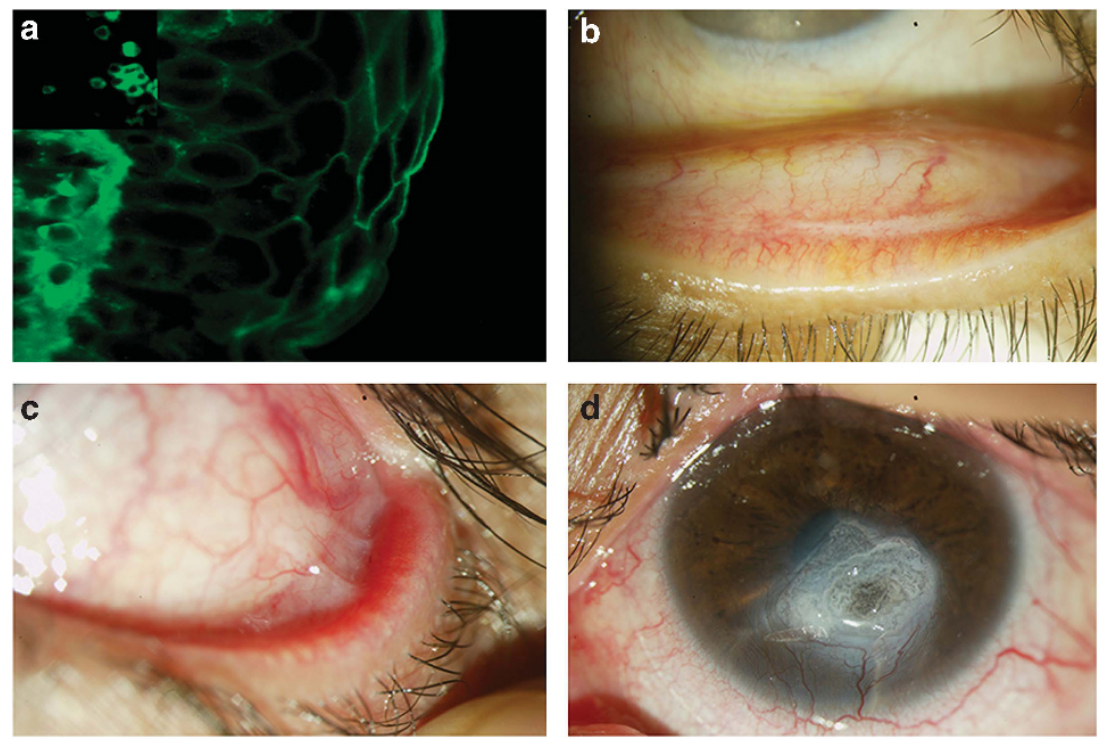

Figure 1 (a) Immunofluorescence: Positive staining in affected conjunctiva showing honeycomb-shaped IgG in the intercellular area (Micrograph $\times 400$ ). Upper micrograph. A positive staining control against human IgG was performed in a human lymph-node tissue from a healthy donor; plasma cells showed intracytoplasmic-positive staining. (b) Stage I: Subconjunctival fibrosis located at the inferior tarsus. (c) Stage III: Fornix foreshortening and symblepharon. (d) Stage IV: Corneal perforation treated with cyanoacrylate patch. 
Table 1 shows demographics, concomitant diseases, and concomitant medications of patients with PV at their initial presentation.

The mean follow-up was 30.8 \pm 25.18 months (range 2-68 months). Of the 15 patients, $1(6.7 \%)$ had characteristic PV lesions on the scalp and oral mucosa and had been diagnosed by a dermatologist in the past. During the follow-up period, two patients (13.3\%) developed blisters on the oral mucosa, which were not present at the onset of ocular involvement.

\section{Concomitant disease}

We found concomitant ocular disease in eight patients (53.3\%): cataracts in six eyes (20\%), glaucoma in six eyes $(20 \%)$, and age-related macular degeneration in four eyes $(13.3 \%)$. Previous ocular surgery was reported in six patients (20\%): cataract extraction in six eyes,

Table 1 Demographics, concomitant ocular and systemic diseases, and medications at initial evaluation

\begin{tabular}{|c|c|c|}
\hline \multicolumn{3}{|l|}{ Sex } \\
\hline Female & 12 & $80 \%$ \\
\hline Male & 3 & $20 \%$ \\
\hline Age (mean) & 68.27 years & \pm 14.35 \\
\hline Previous diagnosis of pemphigus vulgaris & 1 & $6.7 \%$ \\
\hline \multicolumn{3}{|l|}{ Concomitant systemic diseases } \\
\hline Systemic arterial hypertension & 9 & $60 \%$ \\
\hline Diabetes mellitus & 3 & $20 \%$ \\
\hline Hypothyroidism & 1 & $6.7 \%$ \\
\hline Hyperthyroidism & 1 & $6.7 \%$ \\
\hline Sjögren's syndrome & 2 & $13.3 \%$ \\
\hline Rheumatoid arthritis & 1 & $6.7 \%$ \\
\hline Asthma & 1 & $6.7 \%$ \\
\hline \multicolumn{3}{|l|}{ Systemic medications } \\
\hline ACE inhibitors & 4 & $26.7 \%$ \\
\hline Beta-blockers & 3 & $20 \%$ \\
\hline Glibenclamide & 3 & $20 \%$ \\
\hline Angiotensin II receptor blockers & 1 & $6.7 \%$ \\
\hline NSAIDs & 1 & $6.7 \%$ \\
\hline Methimazole & 1 & $6.7 \%$ \\
\hline \multicolumn{3}{|l|}{ Concomitant ocular diseases ${ }^{\mathrm{a}}$} \\
\hline Cataract & 6 & $20 \%$ \\
\hline Glaucoma & 6 & $20 \%$ \\
\hline Age-related macular degeneration & 4 & $13.3 \%$ \\
\hline \multicolumn{3}{|l|}{ Previous ocular surgery ${ }^{a}$} \\
\hline Extracapsular-cataract extraction (ECCE) & 6 & $20 \%$ \\
\hline Trabeculectomy & 4 & $13 \%$ \\
\hline Pterygium resection & 3 & $10 \%$ \\
\hline \multicolumn{3}{|l|}{ Topical medication ${ }^{\mathrm{a}}$} \\
\hline NSAIDs & 2 & $6.7 \%$ \\
\hline Beta-blockers & 3 & $10 \%$ \\
\hline Carbonic anhydrase inhibitors & 5 & $16.7 \%$ \\
\hline Prostaglandin analog & 3 & $10 \%$ \\
\hline
\end{tabular}

${ }^{\text {a }}$ Number of eyes was considered. trabeculectomy in four eyes, and pterygium resection in three eyes, and the mean time between the surgery and their initial examination for the present illness was $3.36 \pm 3.30$ years ( $R: 8.4$ months to 10 years). Diagnoses of systemic disease were found in 10 patients $(66.7 \%)$ : arterial hypertension, in 9 patients $(60 \%)$; diabetes mellitus, in 3 patients (20\%); autoimmune thyroid disease, in 2 patients (13.3\%); Sjögren's syndrome, in 2 patients (13.3\%); and rheumatoid arthritis, in 1 patient (6.7\%).

\section{Concomitant medication}

Five eyes of three patients had history of topical antiglaucomatous agents, three received both topical betablockers and carbonic anhydrase inhibitors (Cosopt; Merck \& Co., West Point, PA, USA) and two eyes only topical carbonic anhydrase inhibitors (Trusopt; Merck \& Co.) and prostaglandin analogs (Travatan (Alcon, Forthworth, TX, USA), Xalatan (Pharmacia \& Upjohn Company, Kalamazoo, MI, USA)) at initial evaluation. Four of these eyes $(80 \%)$ showed progression despite suspension of mentioned medication.

Ten patients $(66.7 \%)$ were exposed to systemic medication associated with pemphigus development (Table 1), and three of them were exposed to two drugs. The most frequent potential pemphigus-inducing drugs used were ACE inhibitors, beta-blockers, and glibenclamide.

\section{Clinical Characteristics}

All of the patients studied reported irritation, burning, redness, or tearing of the eyes as ocular complaints. Ten $(66.7 \%)$ reported a decrease in vision. All of the patients had at least one sign of chronic inflammation and scarring on the conjunctiva (Table 2).

\section{Severity of ocular disease}

According to Foster's classification, ocular involvement was categorized as stage I (6 eyes), stage II (12 eyes), stage III (10 eyes), or stage IV (2 eyes). See Table 2 and Figures $1 \mathrm{~b}-\mathrm{d}$.

The symmetry of ocular involvement was compared between the eyes of the same patient, and a statistically significant bilateral concordance was found ( $R=0.84$, $P=0.0001)$.

At the last follow-up visit, eight eyes of seven patients (46.7\%) showed progression according to Foster's classification. In two eyes, trichiasis and ectropion lead to chronic epithelial defects. The other 22 eyes showed remission of the disease with systemic immunosuppressive treatment. 
Table 2 Frequency and severity of ocular signs in patients with pemphigus vulgaris

\begin{tabular}{lcc}
\hline Ocular signs & No. of eyes & $\%$ \\
\hline Conjunctival hyperemia & 30 & 100 \\
Subconjunctival scarring & 30 & 100 \\
Cul-de-sac shortening & 22 & 73.3 \\
Eyelids (trichiasis or entropion) & 9 & 30 \\
Symblepharon & 12 & 40 \\
Central corneal opacities & 13 & 43.3 \\
Peripheral corneal opacities & 3 & 10 \\
Ankyloblepharon & 2 & 6.7 \\
Severity & & \\
$\quad$ Stage I & 6 & 20 \\
Stage II & 12 & 40 \\
Stage III & 10 & 33 \\
Stage IV & 2 & 7 \\
\hline
\end{tabular}

Corneal perforation was observed as the initial manifestation in three eyes presumably secondary to a persistent epithelial defect. Despite medical treatment, during the follow-up period ( 1 month), perforation in one eye secondary to sicca syndrome was seen.

One of these patients was treated with multiple $N$-butyl2-Cyanoacrylate glue (Histoacryl; B. Braun Melsungen AG, Melsungen, Germany) patches and three of these patients were treated with penetrating keratoplasty.

\section{Visual acuity at diagnosis}

The mean UCVA was logMAR 1.54 (SD 1.14), and the mean BCVA was logMAR 1.46 (SD 1.20), with a range that varied from logMAR $0(20 / 20)$ to $\log$ MAR 4 (NLP).

Excluding the eyes with visual loss due to concomitant ocular diseases, and therefore only eyes with lesions attributable to pemphigus (17 eyes, 56.7\%), the mean BCVA was logMAR 1.66 (20/914), with a range from logMAR $0(20 / 20)$ to $\log$ MAR 4 (NLP).

\section{Specific treatment}

Twelve patients (80\%) had systemic treatment. Prednisone and dapsone were the immunosuppressive agents most frequently prescribed in this series. The other medications used to treat the disease included methotrexate, cyclophosphamide, and azathioprine (Table 3).

Seven patients $(46.7 \%)$ required more than one systemic medication to control the disease. The mean time between conjunctival biopsy and treatment initiation was $83.5 \pm 47.1$ days. Despite systemic treatment, six patients $(50 \%)$ show progression of the disease.
Table 3 Specific systemic treatment established after immunological diagnosis of PV

\begin{tabular}{lcc}
\hline Specific systemic treatment & No. of patients & $\%$ \\
\hline Prednisone & 8 & 53.3 \\
Dapsone & 7 & 46.7 \\
Methotrexate & 4 & 26.7 \\
Azathioprine & 3 & 20 \\
Cyclophosphamide & 3 & 20 \\
\hline
\end{tabular}

\section{Discussion}

The present study represents the largest series of pemphigus patients with ocular involvement diagnosed by the immunopathology of conjunctival samples.

Pemphigus is an autoimmune blistering disease in which IgG antibodies are directed against desmogleins (desmosomal glycoproteins), leading to cell-from-cell detachment and acantholysis. 6,14,15

In classical reports of PV, ocular involvement has been reported as uncommon, not progressive to scarring and readily controlled by systemic immunosuppression. ${ }^{6-8,14-15}$ Typically, conjunctivitis and blepharitis are the main features of the disease, and blisters or erosions on the conjunctiva and the eyelid margin occur but are rare. ${ }^{9,10,16-19}$ Ocular involvement has been reported as a signal of severe disease and tends to occur several months after the onset of skin or other mucosal lesions. ${ }^{6}$ However, there is evidence to suggest that ocular symptoms can precede the onset of skin and mucus membrane lesions, ${ }^{11,20-22}$ suggesting that ocular involvement may be underdiagnosed. In their series of 11 patients with ocular PV, Hodak et al ${ }^{9}$ reported that eight patients $(72.7 \%)$ had symptoms of ocular irritation, tearing, and foreign body sensation before the development of oral or cutaneous lesions. Similarly, in our study, all of the patients referred to the ocular symptoms as their principal complaints, and 14 of 15 patients had no extraocular lesions attributable to PV.

Daoud et $a l^{6}$ reported no changes in visual acuity and no long-term sequelae due to ocular involvement by PV. These findings and those reported by Palleschi et al would suggest a good prognosis for ocular involvement in PV. However, there have been reports of keratolysis, corneal perforation, and eyelid abnormalities (entropion and trichiasis) in patients with extraocular diagnoses of PV and PF, suggesting the presentation of conjunctival scarring in some patients. ${ }^{11,12}$ In our study, all of the patients showed ocular cicatricial changes associated with low visual acuity, and corneal perforation was observed in four patients.

Although, in general, we did not observe any compromise of the skin and other mucosae, the symmetry of ocular involvement suggests systemic or 
environmental factors or bilateral ocular exposure to a triggering agent. Therefore, associations with autoimmune or drug-induced diseases should be considered. We recorded autoimmune diseases in five patients (33.3\%): two with thyroid autoimmune disease, two with Sjögren's syndrome, and one with rheumatoid arthritis. Several reports have described associations between pemphigus and other autoimmune diseases, particularly thymoma, rheumatoid arthritis, systemic lupus erythematous, Sjögren's syndrome, autoimmune thyroid disease, and malignancies. ${ }^{23-29}$ Also coexistence with MMP has been reported. ${ }^{30}$ Sami et al ${ }^{31}$ described six patients with clinical, histological, and immunopathological features of mucous membrane (cicatricial) pemphigoid who failed to respond to conventional immunosuppressive therapy. These patients also showed the presence of IgG4 subclass antibodies against intercellular cement substance, which is a characteristic feature associated with PV. They propose that in these patients, a dual diagnosis should be considered and confirmed by various serological assays and that the presence of two pathogenic autoantibodies in these patients could have contributed to the lack of response to conventional immunosuppressive therapy. The patient series presented here showed characteristic immunopathology of PV on a single conjunctival sample, so that a detailed serological study and maybe a secondary confirmatory biopsy could be necessary to elucidate the coexistence of autoimmune diseases, specially MMP.

Regarding medications, in our series, 10 patients (66.7\%) were exposed to potential pemphigus-inducing drugs. This proportion could be higher because of the retrospective character of the present study and the possible underreporting of the use of potential pemphigus-inducing drugs. In the literature, there are increasing numbers of reports of drug-induced pemphigus. ${ }^{32-38}$ Topical and systemic medications have been suspected, and three groups of chemical structures can cause pemphigus: sulfhydryl radicals (thiol drugs or SH drugs), phenol drugs, and non-thiol non-phenol drugs (Table 4). Thiol and phenol drugs can cause acantholytic changes in the skin. The proposed mechanisms for these include the inhibition of enzymes that aggregate keratinocytes and activation of enzymes, such as plasminogen activator, which disaggregate keratinocytes, among others. Calcium channel blockers can cause pemphigus because calcium is needed for the activity of enzymes that have roles in keratogenesis, and desmogleins are calcium dependent. ${ }^{39-43}$

Penicillamine the prototype of thiol drugs was the first drug reported to induce pemphigus. It has been estimated that $7 \%$ of patients taking this drug for at least 6 months develop pemphigus. For other related
Table 4 Drugs related to pemphigus ${ }^{37-39}$

Thiol drugs or SH drugs
Captopril
Penicillamine
Thiamazole
Gold sodium thiomalate
Phenol drugs
Aspirin
Rifampin
Levodopa
Heroin
Non-thiol non-phenol drugs
NSAIDs
ACE Inhibitors
Calcium channel blockers
Glibenclamide
Dipyrone
Miscellaneous
Penicillin
Cephalosporins
Angiotensin II receptor blockers
Beta-blockers
Progesterone

medications, the frequency of drug-induced pemphigus is unknown. ${ }^{44}$ In our series, progression despite medical treatment may be related to the fact that some of these medications were not stopped after the diagnosis of PV.

We postulate the existence of a group of patients with atypical pemphigus, characterized by ocular involvement as the first manifestation and an aggressive course similar to that of a pemphigoid of the mucous membranes. Why the presentation begins in the eye is unclear, but in this group, other systemic autoimmune diseases, concomitant ocular diseases requiring the chronic use of topical drugs (eg, antiglaucomatous agents), previous ocular surgery, polypharmacy, drug toxicity, inaccessibility to health services and other factors not assessed in this study as UV and occupational exposure might influence the onset, severity and progression of ocular involvement. Specific systemic pharmacotherapy did not achieve the stabilization of the disease in half of the cases. Because of the atypical presentation, specific medical treatment was delayed.

Finally, some limitations of our study need to be considered. Because the retrospective nature, compliance, and associations with other autoimmune diseases and the influence of environmental factors associated (viral infections, thermal burns, ionizing rays, contact allergens, and dietary $)^{44}$ could not properly assessed. Having been conducted at a tertiary ophthalmological referral center, selection bias may be also present, and this series may represent a subgroup of patients with more severe and end-stage disease. 
Therefore, this study may not reflect the real prevalence in the general population.

In summary, patients with ocular involvement due to pemphigus may present at ophthalmological practices, even in the absence of dermatological lesions. The ocular signs in PV patients can vary greatly, ranging from conjunctival hyperemia to loss of corneal transparency. The coexistence of other systemic diseases, particularly autoimmune diseases, and the drug use in cases of atypical presentation of ocular pemphigus should be assessed in the presence of cicatricial changes.

\section{Summary}

What was known before

- Ocular pemphigus showed no cicatricial changes.

\section{What this study adds}

- Largest series of ocular pemphigus with cicatricial changes.

- May be influenced by concomitant autoimmune diseases or drugs.

\section{Conflict of interest}

The authors declare no conflict of interest.

\section{Acknowledgements}

We would like to thank C Guadalupe Quintero for her assistance with pathologic specimens, and Dr Mark Mannis for his critical review of the paper.

\section{References}

1 Jordan E, Scott BS, Razzaque AA. The blistering diseases. Med Clin North Am 1998; 82: 1239-1283.

2 Simon DG, Krutchkoff D, Kaslow RA, Zarbo R. Pemphigus in Hartford County, Connecticut, from 1972 to 1977. Arch Dermatol 1980; 116: 1035-1037.

3 Stanley JR, Amagai M. Pemphigus, bullous impetigo, and the staphylococcal scalded-skin syndrome. $N$ Engl J Med 2006; 355-1800.

4 Becker BA, Gaspari AA. Pemphigus vulgaris and vegetans: bullous diseases. Dermatol Clin 1993; 11: 429-452.

5 Burnham TK. Indirect cutaneous immunofluorescence. J Clin Pathol 1973; 26: 268-272.

6 Daoud YJ, Cervantes R, Foster CS, Ahmed AR. Ocular pemphigus. J Am Acad Dermatol 2005; 53: 585-590.

7 Palleschi GM, Giomi B, Fabbriet P. Ocular involvement in pemphigus. Am J Ophthalmol 2007; 144: 149-152.

8 Smith RJ, Manche EE, Mondino BJ. Ocular cicatricial pemphigoid and ocular manifestations of pemphigus vulgaris. Int Ophthalmol Clin 1997; 37: 63-75.
9 Hodak E, Kremer I, David M, Hazaz B, Rothem A, Feuerman $\mathrm{P}$ et al. Conjunctival involvement in pemphigus vulgaris: a clinical, histopathological and immunofluorescence study. Br J Dermatol 1990; 123: 615-620.

10 Lifshitz T, Levy J, Cagnano E, Halevy S. Severe conjunctival and eyelid involvement in pemphigus vulgaris. Int Ophthalmol 2004; 25: 73-74.

11 Baykal HE, Pleyer U, Sonnichsen K, Thiel HJ, Zierhut M. Severe eye involvement in pemphigus vulgaris. Ophthalmologe 1995; 92: 854-857.

12 Suami M, Kato M, Koide K, Usami Y, Hata N, Machida H et al. Keratolysis in a patient with pemphigus vulgaris. Br J Ophthalmol 2001; 85: 1263.

13 Foster CS, Wilson LA, Elkins LB. Immunosuppressive therapy for progressive ocular cicatricial pemphigoid. Ophthalmology 1982; 89: 340-353.

14 Huilgol SC, Black MM. Management of the immunobullous disorders II Pemphigus. Clin Exp Dermatol 1995; 20: 283-293.

15 Laforest C, Huilgol SC, Casson R, Selva D, Leibovitch I. Autoimmune bullous. Ocular manifestations and management. Drugs 2005; 65(13): 1767-1779.

16 Korman N. Pemphigus. J Am Acad Dermatol 1988; 18: 1219.

17 Camisa C, Meisler DM. Immunobullous diseases with ocular involvement. Dermatol Clin 1992; 10: 555-570.

18 Merchant S, Weinstein M. Pemphigus vulgaris: the eyes have it. Pediatrics 2003; 112: 183-185.

19 Bean SF, Holubar K, Gillett RB. Pemphigus involving the eyes. Arch Dermatol 1975; 111: 1484-1486.

20 Bedell AJ. Ocular pemphigus: a clinical presentation of kodachromes. Trans Am Ophthalmol Soc 1964; 62: 109-122.

21 Bystryn JC, Rudolph JL. Pemphigus. Lancet 2005; 366: 61-73.

22 Yeh SW, Ahmed B, Sami N, Razzaque Ahmed A. Blistering disorders: diagnosis and treatment. Dermatol Ther 2003; 16: 214-223.

23 Falk ES. Pemphigus foliaceus in a patient with rhematoid arthritis and Sjogrens syndrome. Dermatologica 1979; 158: 348-354.

24 Diaz LA, Glamb RW, Silva Jr J. A syndrome of multiple immune autoreactivity. Arch Dermatol 1980; 116: 77-79.

25 Gholizadeh N, Zenouz A, Eslami H. Pemphigus vulgaris associated with rheumatoid arthritis in a patient no taking penicillamine. JODD 2012; 6(1): 33-35.

26 Levine L, Bernstein JE, Soltani K, Medenica MM, Yung CW. Coexisting childhood pemphigus foliaceus and Graves disease. Arch Dermatol 1982; 118: 602-604.

27 Wolf R, Feuerman EJ. Pemphigus in association with autoimmune thyroid disease. Cutis 1981; 27: 423-424.

28 Leonhardt J, Heymann W. Thyroid disease and the skin. Dermatol Clin 2002; 20: 473-481.

29 Bartalena L, Bogazzi F, Chiovato L, Tanda ML, Martino E. Graves' hyperthyroidism and ophthalmopathy associated with pemphigus vulgaris: onset of thyroid autoimmune disease during chronic low-dose glucocorticoid therapy. J Endocrinol Invest 1997; 20: 155-157.

30 Buhac J, Bhol K, Padilla Jr T, Foster CS, Ahmed AR. Coexistence of pemphigus vulgaris and ocular cicatricial pemphigoid. J Am Acad Dermatol 1996; 34(5 Pt 2): 884-886.

31 Sami N, Bhol KC, Beutner EH, Plunkett RW, Leiferman KM, Foster CS et al. Simultaneous presence of mucous membrane pemphigoid and pemphigus vulgaris: molecular characterization of both autoantibodies. Clin Immunol 2001; 100(2): 219-227. 
32 Brenner S, Ruocco V. D-penicillamine-induced pemphigus foliaceus with autoantibodies to desmoglein-1. J Am Acad Dermatol 1998; 39: 137-138.

33 Brenner S, Bialy-Golan A, Crost N. Dipyrone in the induction of pemphigus. J Am Acad Dermatol 1997; 36: 488-490.

34 Matz H, Bialy-Golan A, Brenner S. Diclofenac: a new trigger of pemphigus vulgaris? Dermatology 1997; 195: 48-49.

35 Brenner S, Ruocco V, Bialy-Golan A, Tur E, Flaminio C, Ruocco E et al. Pemphigus and pemphigoid-like effects of nifedipine on in vitro cultured normal human skin explants. Int J Dermatol 1999; 38: 36-40.

36 Goldberg I, Kashman Y, Brenner S. The induction of pemphigus by phenol drugs. Int J Dermatol 1999; 38: 888-892.

37 Kaplan RP, Potter TS, Fox JN. Drug-induced pemphigus related to angiotensin-converting enzyme inhibitors. J Am Acad Dermatol 1992; 26: 364-366.

38 Goldberg I, Sasson A, Gat A, Srebrnik A, Brenner S. Pemphigus vulgaris triggered by glibenclamide and cilazapril. Acta Dermatovenerol Croat 2005; 13: 153-155.
39 Brenner S, Goldberg I. Drug-induced pemphigus. Clin Dermatol. 2011; 29: 455-457.

40 Ruocco V, De Angelis E, Lombardi ML. Drug-induced pemphigus. II. Pathomechanisms and experimental investigations. Clin Dermatol 1993; 11: 507-513.

41 Bae YI, Yun SJ, Lee SC, Park GT, Lee JB. Pemphigus foliaceus induced by an angiotensin II receptor blocker. Clin Exp Dermatol 2008; 33(6): 721-723.

42 Newby CS, Barr RM, Greaves MW, Mallet AI. Cytokine release and cytotoxicity in human keratinocytes and fibroblasts induce d by phenols and sodium dodecyl sulfate. J Invest Dermatol 2000; 115: 292-298.

43 Feliciani C, Toto P, Amerio P, Pour SM, Coscione G, Shivji G et al. In vitro and in vivo expression of interleukin- $1 \alpha$ and tumor necrosis factor- $\alpha$ mRNA in pemphigus vulgaris: interleukin- $1 \alpha$ and tumor necrosis factor- $\alpha$ are involved in acantholysis. J Invest Dermatol 2000; 114: 71-77.

44 Ruocco V, Ruocco E. Pemphigus and environmental factors. G Ital Dermatol Venereol 2003; 138: 299-309. 